Document downloaded from:

http://hdl.handle.net/10251/85287

This paper must be cited as:

Martínez Casas, J.; Fayos Sancho, J.; Denia Guzmán, FD.; Baeza González, LM. (2012). Dynamics of damped rotating solids of revolution through an Eulerian modal approach. Journal of Sound and Vibration. 331(4):868-882. doi:10.1016/j.jsv.2011.10.003.

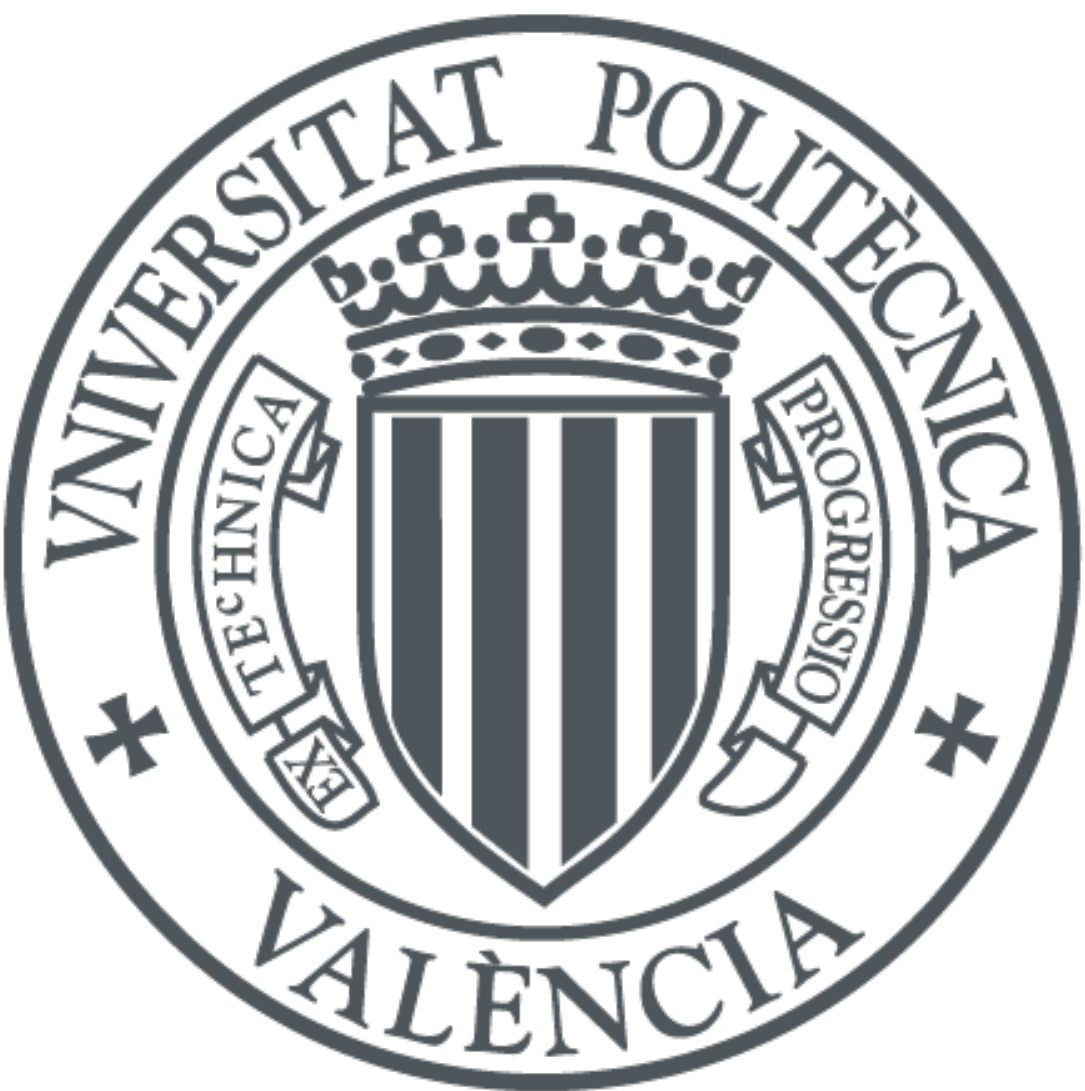

The final publication is available at

http://dx.doi.org/10.1016/j.jsv.2011.10.003

Copyright Elsevier

Additional Information 


\title{
DYNAMICS OF DAMPED ROTATING SOLIDS OF REVOLUTION THROUGH AN EULERIAN MODAL APPROACH
}

\author{
José Martínez-Casas ${ }^{1}$, Juan Fayos ${ }^{2}$, Francisco D. Denia ${ }^{1}$ and Luis Baeza ${ }^{1 *}$ \\ ${ }^{1}$ Centro de Investigación de Tecnología de Vehículos, Universidad Politécnica de Valencia, \\ Camino de Vera s/n, 46022 Valencia, Spain. \\ ${ }^{2}$ Instituto de Biomecánica de Valencia, Universidad Politécnica de Valencia, Camino de Vera \\ s/n, 46022 Valencia, Spain. \\ *E-mail contact address: Lbaeza@mcm.upv.es
}

\begin{abstract}
This article presents a technique for modelling the dynamic response of rotating flexible solids with internal modal damping. The method is applicable to solids with geometry of revolution that rotate around their main axes at constant spinning velocity. The model makes use of an Eulerian modal coordinate system which adopts the vibration modes in a non-rotating frame as basis functions. Due to the coordinate system, the technique is particularly suitable for studying the dynamic interaction between rotating solids and non-rotating structures and permits to obtain Frequency Response Functions. The approach has been adopted to study the dynamics of a simplysupported cylinder in order to obtain the receptance function and the modal properties of the rotating solid.
\end{abstract}

\section{Introduction}

The dynamics of damped shafts was investigated in early works at the first decades of the twentieth century [1,2]. The unstabilising consequence of the internal damping is a well-known phenomenon that produces a critical angular velocity above which the rotor becomes unstable (see monographs in [3,4]). The literature shows many articles that analyse internal damping in Jeffcott rotors and rotating beams but few reported attempts have been found regarding the dynamic models for generic geometries.

The model of elastic rotating solids with generic geometry has to define different shaft section properties (e.g. axles with non-constant cross-sections) and sometimes it needs to be based on a three dimensional domain (e.g. railway wheelsets; Jeffcott rotors and rotating beams are based on a zero and one dimensional domain respectively). The 
Finite Element (FE) Method is possibly the numerical approach that can be more easily adapted to a generic geometry. FE has been adopted through beam elements [5-10], and 3D solid elements [11-13]. The main inconveniences of this technique are found when the solid interacts with a non-rotating structure. The force that the non-rotating system exerts on the rotating one is applied at fixed spatial points, and consequently there is a relative motion between the load and the rotating solid (moving load problem). This fact requires the computation of the contribution of the external force to the generalised force term in each integration step during the simulation. Furthermore the Frequency Response Function (FRF) that correlates the steady response to harmonic excitation produced by a non-mobile force cannot be obtained directly. In addition, a constant and non-mobile force applied on an external surface of a rotating mesh produces a parametric excitation because the force has different effect if it is applied in a node or in the middle of a FE solid. The Jeffcott rotor model and the rotating beam model solve this difficulty because they are based on a zero-dimensional and one-dimensional domain respectively. Moreover, the displacements and velocities of these models are obtained in rotating or non-rotating coordinates indistinctly.

The work presented in [14] proposed a method that solved the above mentioned problems. The method is adequate for undamped solids with geometry of revolution, and it is based on Eulerian modal coordinates. Eulerian coordinates are frequently used in Fluid Mechanics, and they relate spatial points through a fixed coordinate frame. The technique exploits the properties of the solids of revolution whose vibration modes do not depend on the rotation of the system. Consequently the vibration modes of the solid in a fixed coordinate frame are used as basis function in order to define a generic displacement of the flexible solid.

The present work develops a methodology based on Ref. [14] for modelling the dynamic behaviour of rotating solids of revolution with internal modal damping. Results of the proposed model are presented for a simply supported rotating cylinder. This simple geometry provides a reference solution which permits to compare the analytical formulation by means of the rotating Rayleigh beam theory with the results from the proposed method. The formulation associated with damped rotating beams which are needed in this article was developed in [3] and can be found in the Appendix.

Section 2 presents the development of the equation of motion associated with the proposed model. A modal approach is carried out and consequently the modal properties of the non-rotating solid need to be obtained. Section 3 builds the matrices of the equation of motion from the analytical modes of the simply supported Rayleigh beam. At this point, the differences of the new formulation in comparison with the wellestablished methods (like spinning beam theories) can be found.

The proposed methodology can only be formulated analytically in simple cases, e.g. beam models. In Section 4, a numerical methodology is given to analyse more complex structures such as railway wheelsets, non-vented disc brakes or other solids with generic geometry of revolution. Given that theoretical modal analysis of general structures is usually made with the finite element method, this technique is adapted in this paper to obtain the matrices associated with the equation of motion.

The results are present in Section 5 of this article, where FRF of the rotating cylinder and the stability analysis is carried out. The conclusions of this paper are discussed in Section 6. 


\section{Proposed model}

The development of the proposed model is based on a previous work from Brown and Shabana [15]. This work makes use of the floating frame of reference, which is a coordinate system described in detail in Ref. [16]. The proposed method develops an Eulerian modal coordinate system, and the present Section shows its properties and a procedure to derive this coordinate system from the floating frame of reference. This mathematical development leads the equation of motion from the model presented in [15] through a change of variables.

\subsection{Coordinate reference set and its properties}

\subsubsection{Floating frame of reference}

The coordinate system proposed by Shabana [16] obtains the global position of a flexible solid as a sum of two different displacement types. The former can be considered as a rigid body displacement, while the latter corresponds to the displacements due to the deformation of the solid. In order to develop the model of a solid of revolution, the method considers two reference frame systems (see Fig. 1): a fixed frame $\mathbf{X Y Z}$, where the $\mathbf{Z}$-axis is the rotation axis; and a mobile frame $\mathbf{X}^{\prime} \mathbf{Y} \mathbf{Z}^{\prime}$ which rotates at the constant angular velocity of the solid $\Omega$ and it is fixed with the undeformed solid; $\mathbf{X Y Z}$ and $\mathbf{X}^{\prime} \mathbf{Y}^{\prime} \mathbf{Z}$ ' coincide at instant $t=0$. A vector referred to the fixed and the mobile frames is denoted by $\mathbf{a}$ and $\mathbf{a}^{\prime}$ respectively. The position vector $\mathbf{r}$ of a material particle of the solid in relation to the origin of the fixed frame can be expressed as follows

$$
\mathbf{r}=\mathbf{A}\left(\mathbf{u}^{\prime}+\boldsymbol{\Phi}\left(\mathbf{u}^{\prime}\right) \mathbf{p}(t)\right)
$$

where $\mathbf{u}^{\prime}$ is the position vector of the particle in the undeformed configuration, the product $\boldsymbol{\Phi}\left(\mathbf{u}^{\prime}\right) \mathbf{p}(t)$ corresponds to the displacement of the particle due to the elastic deformation of the solid in the rotating frame, $\boldsymbol{\Phi}\left(\mathbf{u}^{\prime}\right)$ contains the mass-normalised mode shapes of the non-rotating solid, $\mathbf{p}(t)$ is the vector of modal coordinates and $\mathbf{A}$ is the rotation matrix, defined by

$$
\mathbf{A}=\left(\begin{array}{ccc}
\cos \theta & -\sin \theta & 0 \\
\sin \theta & \cos \theta & 0 \\
0 & 0 & 1
\end{array}\right)=\left(\begin{array}{ccc}
\cos \Omega t & -\sin \Omega t & 0 \\
\sin \Omega t & \cos \Omega t & 0 \\
0 & 0 & 1
\end{array}\right)
$$

$\theta=\Omega t$ being the angle of rotation. 


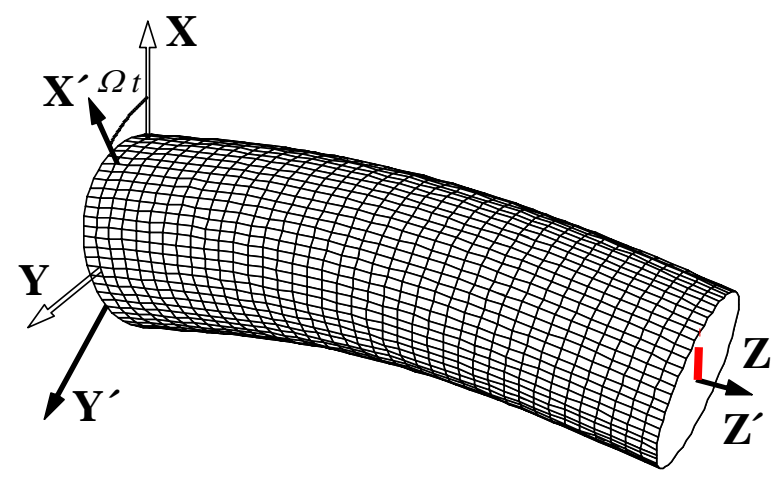

Fig. 1. Coordinate frame systems.

\subsubsection{Eulerian modal coordinates}

It must be highlighted that the mode shape matrix $\boldsymbol{\Phi}$ is computed for the nonrotating solid. In general geometries, they can only be used to define the displacements due to the deformation if the modes are computed in a frame associated with the solid, that is, $\boldsymbol{\Phi}\left(\mathbf{u}^{\prime}\right)$. The properties of the solids of revolution allow to obtain any deformed geometry of the solid through the mode shapes in non-rotating coordinates as follows. Let $\mathbf{v}$ be the position vector of a spatial point in relation to the origin of the fixed frame. The vector $\mathbf{v}$ is defined in the undeformed domain or the volume occupied by the undeformed solid. The position vector of a particle of the solid that occupies the spatial position $\mathbf{v}$ in the undeformed configuration at the instant $t$ verifies

$$
\mathbf{v}=\mathbf{A} \mathbf{u}^{\prime} .
$$

The position of the particle after deformation is

$$
\mathbf{r}=\mathbf{v}+\boldsymbol{\Phi}(\mathbf{v}) \mathbf{q}(t)
$$

if the particle occupies the spatial position $\mathbf{v}$ in the undeformed configuration, where $\mathbf{q}(t)$ is defined the Eulerian-modal coordinate vector.

From Eqs. (1) and (4), it follows

$$
\mathbf{\Phi}(\mathbf{v}) \mathbf{q}(t)=\mathbf{A} \mathbf{\Phi}\left(\mathbf{u}^{\prime}\right) \mathbf{p}(t) .
$$

Eq. (5) is multiplied by $\rho \boldsymbol{\Phi}(\mathbf{v})^{\mathrm{T}}$, where $\rho$ is the density, and an integration is then carried out over the volume of the solid. Due to the orthogonality of the modes, the integral yields

$$
\mathbf{q}(t)=\left(\iiint_{\text {Volume }} \rho \boldsymbol{\Phi}(\mathbf{v})^{\mathrm{T}} \mathbf{A} \boldsymbol{\Phi}\left(\mathbf{A}^{\mathrm{T}} \mathbf{v}\right) \mathrm{d} v\right) \mathbf{p}(t) .
$$



follows

Equation [6] provides the coordinate transformation, which can be written as

$$
\mathbf{q}(t)=\mathbf{B}(t) \mathbf{p}(t)
$$

2.1.3 Two basic properties of the integrals on the solid of revolution

Taking into account that the jacobian is equal to 1 , any scalar field $\mu$ integrable on the domain of the solid of revolution has the following property

$$
\iiint_{\text {Volume }} \mu(\mathbf{v}) \mathrm{d} v=\iiint_{\text {Volume }} \mu\left(\mathbf{u}^{\prime}\right) \mathrm{d} v \text {. }
$$

Let us consider the matrix $\tilde{\mathbf{M}}$ which is defined as follows

$$
\tilde{\mathbf{M}}=\iiint_{\text {Volume }} \rho \boldsymbol{\Phi}\left(\mathbf{u}^{\prime}\right)^{\mathrm{T}} \mathbf{M} \boldsymbol{\Phi}\left(\mathbf{u}^{\prime}\right) \mathrm{d} v,
$$

where $\mathbf{M}$ is a constant $3 \times 3$ matrix. If matrix $\mathbf{M}$ verifies that

$$
\mathbf{M}=\mathbf{A}^{\mathrm{T}} \mathbf{M} \mathbf{A},
$$

the transformation matrix $\mathbf{B}(t)$ has no influence if it is applied to the matrix $\tilde{\mathbf{M}}$, that is

$$
\mathbf{B}(t)^{\mathrm{T}} \tilde{\mathbf{M}} \mathbf{B}(t)=\tilde{\mathbf{M}} .
$$

The proof is the following

$$
\begin{aligned}
\mathbf{B}(t)^{\mathrm{T}} \tilde{\mathbf{M}} \mathbf{B}(t) & =\mathbf{B}(t)^{\mathrm{T}}\left(\iiint_{\text {Volume }} \rho \boldsymbol{\Phi}\left(\mathbf{u}^{\prime}\right)^{\mathrm{T}} \mathbf{M} \boldsymbol{\Phi}\left(\mathbf{u}^{\prime}\right) \mathrm{d} v\right) \mathbf{B}(t) \\
& =\iiint_{\text {Volume }} \rho \mathbf{B}(t)^{\mathrm{T}} \boldsymbol{\Phi}\left(\mathbf{u}^{\prime}\right)^{\mathrm{T}} \mathbf{M} \boldsymbol{\Phi}\left(\mathbf{u}^{\prime}\right) \mathbf{B}(t) \mathrm{d} v \\
& =\iiint_{\text {Volume }} \rho \mathbf{B}(t)^{\mathrm{T}} \boldsymbol{\Phi}(\mathbf{v})^{\mathrm{T}} \mathbf{M} \boldsymbol{\Phi}(\mathbf{v}) \mathbf{B}(t) \mathrm{d} v \\
& =\iiint_{\text {Volume }} \rho \boldsymbol{\Phi}\left(\mathbf{u}^{\prime}\right)^{\mathrm{T}} \mathbf{A}^{\mathrm{T}} \mathbf{M} \mathbf{A} \boldsymbol{\Phi}\left(\mathbf{u}^{\prime}\right) \mathrm{d} v \\
& =\iiint_{\text {Volume }} \rho \boldsymbol{\Phi}\left(\mathbf{u}^{\prime}\right)^{\mathrm{T}} \mathbf{M} \boldsymbol{\Phi}\left(\mathbf{u}^{\prime}\right) \mathrm{d} v=\tilde{\mathbf{M}} .
\end{aligned}
$$

2.1.4 Orthogonality of the transformation matrix $\mathbf{B}(t)$ 
The transformation matrix $\mathbf{B}(t)$ can be proved to be orthogonal. If Eq. (7) is substituted into Eq. (5), one obtains

$$
\boldsymbol{\Phi}(\mathbf{v}) \mathbf{B}(t)=\mathbf{A} \boldsymbol{\Phi}\left(\mathbf{A}^{\mathrm{T}} \mathbf{v}\right)
$$

Each side of the last equation is multiplied by itself (transposed) and the density, giving

$$
\rho \mathbf{B}(t)^{\mathrm{T}} \boldsymbol{\Phi}(\mathbf{v})^{\mathrm{T}} \boldsymbol{\Phi}(\mathbf{v}) \mathbf{B}(t)=\rho \boldsymbol{\Phi}\left(\mathbf{A}^{\mathrm{T}} \mathbf{v}\right)^{\mathrm{T}} \mathbf{A}^{\mathrm{T}} \mathbf{A} \boldsymbol{\Phi}\left(\mathbf{A}^{\mathrm{T}} \mathbf{v}\right)
$$

Eq. (14) is now integrated over the solid volume

$$
\mathbf{B}(t)^{\mathrm{T}}\left(\iiint_{\text {Volume }} \rho \boldsymbol{\Phi}(\mathbf{v})^{\mathrm{T}} \boldsymbol{\Phi}(\mathbf{v}) \mathrm{d} v\right) \mathbf{B}(t)=\iiint_{\text {Volume }} \rho \boldsymbol{\Phi}\left(\mathbf{A}^{\mathrm{T}} \mathbf{v}\right)^{\mathrm{T}} \boldsymbol{\Phi}\left(\mathbf{A}^{\mathrm{T}} \mathbf{v}\right) \mathrm{d} v .
$$

Considering now Eq. (15), the property obtained in Eq. (8), and the mode shapes orthogonality, the relation $\mathbf{B}(t)^{\mathrm{T}} \mathbf{B}(t)=\mathbf{I}$ is found and consequently $\mathbf{B}(t)$ is an orthogonal matrix.

\subsubsection{Structure of the transformation matrix $\mathbf{B}(t)$}

Let us consider different $n$-th and $m$-th modes and the corresponding mode shapes $\boldsymbol{\Phi}^{<n>}$ and $\boldsymbol{\Phi}^{<m>}$. Eq. (13) can be written for the $n$-th mode as follows

$$
\boldsymbol{\Phi}(\mathbf{v}) \mathbf{B}^{<n>}=\mathbf{A} \boldsymbol{\Phi}\left(\mathbf{A}^{\mathrm{T}} \mathbf{v}\right)^{<n>} .
$$

Now, Eq. (16) is pre-multiplied by $\rho \boldsymbol{\Phi}^{<m>}$ (transposed), and then integrated on the solid volume, giving

$$
B_{m n}=\iiint_{\text {Volume }} \rho\left(\mathbf{\Phi}^{<m>}\right)^{\mathrm{T}} \mathbf{A} \boldsymbol{\Phi}\left(\mathbf{A}^{\mathrm{T}} \mathbf{v}\right)^{<n>} d v
$$

If the modes $\boldsymbol{\Phi}^{<n>}$ and $\boldsymbol{\Phi}^{<m>}$ are orthogonal, due to the geometry of revolution $\mathbf{A} \boldsymbol{\Phi}\left(\mathbf{A}^{\mathrm{T}} \mathbf{v}\right)^{<n>}$ and $\boldsymbol{\Phi}^{<m>}$ are also orthogonal, and consequently $B_{m n}=0$.

The rows and columns of matrix $\mathbf{B}(t)$ associated with modes with multiplicity 1 have null entries except the diagonal entry, which is equal to 1 . Due to the orthogonality property of $\mathbf{B}(t)$, the diagonal blocks associated with modes with multiplicity 2 contain rotation matrices, that is 


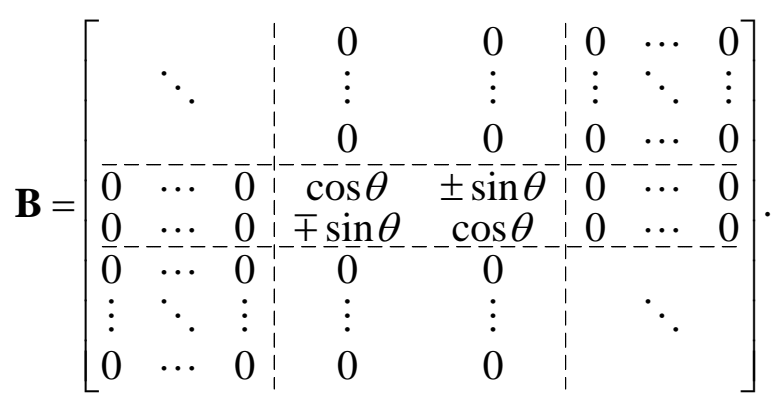

\subsubsection{Additional remark}

Let $\tilde{\mathbf{M}}$ be a diagonal matrix of dimension $N \times N$, where $N$ is the number of degrees of freedom of the solid model. The following relationship

$$
\mathbf{B}(t)^{\mathrm{T}} \tilde{\mathbf{M}} \mathbf{B}(t)=\tilde{\mathbf{M}}
$$

is verified if the pair of entries associated with each mode with multiplicity 2 are equal. The proof is found through the structure of the matrix $\mathbf{B}(t)$ showed in the previous section.

If $\tilde{\mathbf{M}}$ is a column matrix of dimension $N \times 1$, the following relation is fulfilled

$$
\mathbf{B}(t)^{\mathrm{T}} \tilde{\mathbf{M}}=\tilde{\mathbf{M}}
$$

only if the non-zero entries in $\tilde{\mathbf{M}}$ are associated with modes of 1 multiplicity.

\subsection{Development of the equation of motion}

\subsubsection{Damped equation of motion through Lagrangian coordinates}

The method presented in [15] develops the following equation of motion for rotating solids

$$
\ddot{\mathbf{p}}+2 \Omega \tilde{\mathbf{J}} \dot{\mathbf{p}}+\left(\tilde{\mathbf{K}}-\Omega^{2} \tilde{\mathbf{E}}\right) \mathbf{p}=\mathbf{Q}_{\mathbf{p}}+\Omega^{2} \tilde{\mathbf{L}}
$$

The components of Eq. (21) are detailed next. Matrix $\tilde{\mathbf{K}}$ is the modal stiffness matrix, whose diagonal contains the square of the undamped natural frequencies of the solid. Matrix $\tilde{\mathbf{J}}$ is associated with the gyroscopic effect, and it is computed as follows

$$
\tilde{\mathbf{J}}=\iiint_{\text {Volume }} \rho \boldsymbol{\Phi}^{\mathrm{T}} \mathbf{J} \boldsymbol{\Phi} \mathrm{d} v
$$

where 


$$
\mathbf{J}=\mathbf{A}_{\theta} \mathbf{A}^{\mathrm{T}}=\mathbf{A}^{\mathrm{T}} \mathbf{A}_{\theta},
$$

$\mathbf{A}_{\theta}$ being the derivative of the matrix $\mathbf{A}$ with respect to $\theta$, that is

$$
\mathbf{A}_{\theta}=\left(\begin{array}{ccc}
-\sin \theta & -\cos \theta & 0 \\
\cos \theta & -\sin \theta & 0 \\
0 & 0 & 0
\end{array}\right) .
$$

The development of the Eq. (23) shows that

$$
\mathbf{J}=\left(\begin{array}{ccc}
0 & -1 & 0 \\
1 & 0 & 0 \\
0 & 0 & 0
\end{array}\right) .
$$

Some vibration modes produce deformed shapes which may permit centrifugal forces to produce work. This effect is considered through the matrix $\tilde{\mathbf{E}}$ which is calculated as

$$
\widetilde{\mathbf{E}}=\iiint_{\text {Volume }} \rho \boldsymbol{\Phi}^{\mathrm{T}} \mathbf{E} \boldsymbol{\Phi} \mathrm{d} v,
$$

where

$$
\mathbf{E}=-\mathbf{A}_{\theta \theta} \mathbf{A}^{\mathrm{T}}=-\mathbf{A}^{\mathrm{T}} \mathbf{A}_{\theta \theta},
$$

$\mathbf{A}_{\theta \theta}$ being the second derivative of the matrix $\mathbf{A}$ with respect to $\theta$, that is

$$
\mathbf{A}_{\theta \theta}=\left(\begin{array}{ccc}
-\cos \theta & \sin \theta & 0 \\
-\sin \theta & -\cos \theta & 0 \\
0 & 0 & 0
\end{array}\right) .
$$

It can be easily obtained that

$$
\mathbf{E}=\left(\begin{array}{lll}
1 & 0 & 0 \\
0 & 1 & 0 \\
0 & 0 & 0
\end{array}\right) .
$$

The centrifugal forces that do not depend on the solid deformation are implemented by means of $\tilde{\mathbf{L}}$. These forces are associated with modes of multiplicity 1 , and only the entries of $\tilde{\mathbf{L}}$ associated with these modes are non-zero. The column matrix $\tilde{\mathbf{L}}$ is obtained as follows

$$
\tilde{\mathbf{L}}=\iiint_{\text {Volume }} \rho \boldsymbol{\Phi}^{\mathrm{T}} \mathbf{E} \mathbf{u}^{\prime} \mathrm{d} v .
$$


If the external forces are applied in fixed material points, the generalised force term is computed by

$$
\mathbf{Q}_{\mathrm{p}}(t)=\iiint_{\text {Volume }} \boldsymbol{\Phi}\left(\mathbf{u}^{\prime}\right)^{\mathrm{T}} \mathbf{A}(t)^{\mathrm{T}} \mathbf{f}^{v}\left(\mathbf{u}^{\prime}, t\right) \mathrm{d} v,
$$

where $\mathbf{f}^{v}\left(\mathbf{u}^{\prime}, t\right)$ are the external volume forces. However, if the external forces $\mathbf{g}^{v}(\mathbf{v}, t)$ are applied in fixed spatial points, the generalised force term can be calculated from the following expression

$$
\mathbf{Q}_{\mathrm{p}}(t)=\iiint_{\text {Volume }} \mathbf{\Phi}\left(\mathbf{A}(t)^{\mathrm{T}} \mathbf{v}\right)^{\mathrm{T}} \mathbf{A}(t)^{\mathrm{T}} \mathbf{g}^{v}(\mathbf{v}, t) \mathrm{d} v .
$$

Internal modal damping can be implemented in Eq. (21) as detailed next. Let $\widetilde{\mathbf{D}}$ be the diagonal modal damping matrix, where the modes of double multiplicity contains two equal terms. The generalised force due to damping is computed by means of

$$
\mathbf{Q}_{\mathrm{d}}(t)=-\tilde{\mathbf{D}} \dot{\mathbf{p}}
$$

Introducing Eq. (33) into Eq. (21), the equation of motion of the internally damped solid is

$$
\ddot{\mathbf{p}}+(\tilde{\mathbf{D}}+2 \Omega \tilde{\mathbf{J}}) \dot{\mathbf{p}}+\left(\tilde{\mathbf{K}}-\Omega^{2} \tilde{\mathbf{E}}\right) \mathbf{p}=\mathbf{Q}_{\mathbf{p}}+\Omega^{2} \tilde{\mathbf{L}}
$$

\subsubsection{Damped equation of motion through Eulerian coordinates}

The coordinate change expressed by Eq. (7) is introduced in Eq. (34), and then the resulting equation is multiplied by $\mathbf{B}(t)$, giving

$$
\begin{aligned}
& \ddot{\mathbf{q}}+\left(\mathbf{B} \tilde{\mathbf{D}} \mathbf{B}^{\mathrm{T}}+2 \Omega \mathbf{B} \tilde{\mathbf{J}} \mathbf{B}^{\mathrm{T}}+2 \mathbf{B} \dot{\mathbf{B}}^{\mathrm{T}}\right) \dot{\mathbf{q}}+ \\
& +\left(\mathbf{B} \tilde{\mathbf{K}} \mathbf{B}^{\mathrm{T}}-\Omega^{2} \mathbf{B} \tilde{\mathbf{E}} \mathbf{B}^{\mathrm{T}}+\mathbf{B} \ddot{\mathbf{B}}^{\mathrm{T}}+\mathbf{B} \tilde{\mathbf{D}} \dot{\mathbf{B}}^{\mathrm{T}}+2 \Omega \mathbf{B} \tilde{\mathbf{J}} \dot{\mathbf{B}}^{\mathrm{T}}\right) \mathbf{q}=\mathbf{B} \mathbf{Q}_{\mathbf{p}}+\Omega^{2} \mathbf{B} \tilde{\mathbf{L}} .
\end{aligned}
$$
are found

Due to the properties stated in Sections 2.1.3 and 2.1.6, the following equations

$$
\begin{aligned}
\mathbf{B} \tilde{\mathbf{J}} \mathbf{B}^{\mathrm{T}} & =\tilde{\mathbf{J}}, \\
\mathbf{B} \tilde{\mathbf{E}} \mathbf{B}^{\mathrm{T}} & =\widetilde{\mathbf{E}}, \\
\mathbf{B} \tilde{\mathbf{K}} \mathbf{B}^{\mathrm{T}} & =\tilde{\mathbf{K}}, \\
\mathbf{B} \tilde{\mathbf{D}} \mathbf{B}^{\mathrm{T}} & =\tilde{\mathbf{D}}, \\
\mathbf{B} \tilde{\mathbf{L}} & =\tilde{\mathbf{L}} .
\end{aligned}
$$


The calculation of the terms $\mathbf{B} \tilde{\mathbf{D}} \dot{\mathbf{B}}^{\mathrm{T}}$ and $\mathbf{B} \tilde{\mathbf{J}} \dot{\mathbf{B}}^{\mathrm{T}}$ is reduced to the evaluation of $\mathbf{B} \dot{\mathbf{B}}^{\mathrm{T}}$, because $\mathbf{B} \tilde{\mathbf{D}}=\mathbf{B} \tilde{\mathbf{D}} \mathbf{B}^{\mathrm{T}} \mathbf{B}=\tilde{\mathbf{D}} \mathbf{B}$ and $\mathbf{B} \tilde{\mathbf{J}}=\mathbf{B} \tilde{\mathbf{J}} \mathbf{B}^{\mathrm{T}} \mathbf{B}=\tilde{\mathbf{J}} \mathbf{B}$. From Eq. (13),

$$
\mathbf{B} \boldsymbol{\Phi}\left(\mathbf{u}^{\prime}\right)^{\mathrm{T}}=\boldsymbol{\Phi}(\mathbf{v})^{\mathrm{T}} \mathbf{A} .
$$

Eq. (41) is derivated with respect to time,

$$
\dot{\mathbf{B}} \boldsymbol{\Phi}\left(\mathbf{u}^{\prime}\right)^{\mathrm{T}}=\dot{\boldsymbol{\Phi}}(\mathbf{v})^{\mathrm{T}} \mathbf{A}+\boldsymbol{\Phi}(\mathbf{v})^{\mathrm{T}} \dot{\mathbf{A}}
$$

In Eq. (42) the function $\boldsymbol{\Phi}\left(\mathbf{u}^{\prime}\right)$ is constant because it is associated with a material point. On the other hand, $\dot{\boldsymbol{\Phi}}(\mathbf{v})$ has to be computed as the convective term of the material derivative, that is

$$
\begin{aligned}
\dot{\boldsymbol{\Phi}}(\mathbf{v}) & =\sum_{i=1}^{3} \frac{\partial \boldsymbol{\Phi}(\mathbf{v})}{\partial v_{i}} \frac{\mathrm{d} v_{i}}{\mathrm{~d} t}=\sum_{i=1}^{3} \frac{\partial \boldsymbol{\Phi}(\mathbf{v})}{\partial v_{i}} \frac{\mathrm{d}\left(\mathbf{A} \mathbf{u}^{\prime}\right)_{i}}{\mathrm{~d} t} \\
& =\sum_{i=1}^{3} \frac{\partial \boldsymbol{\Phi}(\mathbf{v})}{\partial v_{i}}\left(\Omega \mathbf{A}_{\theta} \mathbf{u}^{\prime}\right)_{i}=\Omega \sum_{i=1}^{3} \frac{\partial \boldsymbol{\Phi}(\mathbf{v})}{\partial v_{i}}(\mathbf{J} \mathbf{v})_{i} .
\end{aligned}
$$

Eq. (42) is now post-multiplied by $\boldsymbol{\Phi}\left(\mathbf{u}^{\prime}\right) \mathbf{B}^{\mathrm{T}}$, yielding

$$
\dot{\mathbf{B}} \boldsymbol{\Phi}\left(\mathbf{u}^{\prime}\right)^{\mathrm{T}} \boldsymbol{\Phi}\left(\mathbf{u}^{\prime}\right) \mathbf{B}^{\mathrm{T}}=\Omega\left(\sum_{i=1}^{3} \frac{\partial \boldsymbol{\Phi}(\mathbf{v})^{\mathrm{T}}}{\partial v_{i}}(\mathbf{J} \mathbf{v})_{i}\right) \mathbf{A} \boldsymbol{\Phi}\left(\mathbf{u}^{\prime}\right) \mathbf{B}^{\mathrm{T}}+\Omega \boldsymbol{\Phi}(\mathbf{v})^{\mathrm{T}} \mathbf{A}_{\theta} \boldsymbol{\Phi}\left(\mathbf{u}^{\prime}\right) \mathbf{B}^{\mathrm{T}}
$$

Recalling the result in Eq. (13), Eq. (44) becomes

$$
\begin{aligned}
\dot{\mathbf{B}} \boldsymbol{\Phi}\left(\mathbf{u}^{\prime}\right)^{\mathrm{T}} \boldsymbol{\Phi}\left(\mathbf{u}^{\prime}\right) \mathbf{B}^{\mathrm{T}} & =\Omega\left(\sum_{i=1}^{3} \frac{\partial \boldsymbol{\Phi}(\mathbf{v})^{\mathrm{T}}}{\partial v_{i}}(\mathbf{J} \mathbf{v})_{i}\right) \boldsymbol{\Phi}(\mathbf{v}) \mathbf{B} \mathbf{B}^{\mathrm{T}}+\Omega \boldsymbol{\Phi}(\mathbf{v})^{\mathrm{T}} \mathbf{A}_{\theta} \mathbf{A}^{\mathrm{T}} \boldsymbol{\Phi}(\mathbf{v}) \mathbf{B} \mathbf{B}^{\mathrm{T}} \\
& =\Omega\left(\sum_{i=1}^{3} \frac{\partial \boldsymbol{\Phi}(\mathbf{v})^{\mathrm{T}}}{\partial v_{i}}(\mathbf{J} \mathbf{v})_{i}\right) \boldsymbol{\Phi}(\mathbf{v})+\Omega \boldsymbol{\Phi}(\mathbf{v})^{\mathrm{T}} \mathbf{J} \boldsymbol{\Phi}(\mathbf{v}) .
\end{aligned}
$$

Now Eq. (45) is multiplied by the density $\rho$ and it is integrated on the volume of the solid. Applying the orthogonality property, yields

$$
\dot{\mathbf{B}} \mathbf{B}^{\mathrm{T}}=\Omega \iiint_{\text {Volume }}\left(\sum_{i=1}^{3} \frac{\partial \boldsymbol{\Phi}(\mathbf{v})^{\mathrm{T}}}{\partial v_{i}}(\mathbf{J} \mathbf{v})_{i}\right) \boldsymbol{\Phi}(\mathbf{v}) \rho \mathrm{d} v+\Omega \tilde{\mathbf{J}} .
$$

Considering now the first property in Section 2.1.3, the integral in the Eq. (46) does not depend on time. Lets define the following variable, that is

$$
\widetilde{\mathbf{G}}=\iiint_{\text {Volume }}\left(\sum_{i=1}^{3} \frac{\partial \boldsymbol{\Phi}\left(\mathbf{u}^{\prime}\right)^{\mathrm{T}}}{\partial u_{i}}\left(\mathbf{J} \mathbf{u}^{\prime}\right)_{i}\right) \boldsymbol{\Phi}\left(\mathbf{u}^{\prime}\right) \rho \mathrm{d} v,
$$

and 


$$
\dot{\mathbf{B}} \mathbf{B}^{\mathrm{T}}=\Omega(\tilde{\mathbf{G}}+\tilde{\mathbf{J}})
$$

The calculation of $\mathbf{B} \ddot{\mathbf{B}}^{\mathrm{T}}$ is performed as follows. The product $\dot{\mathbf{B}} \mathbf{B}^{\mathrm{T}}$ is constant and consequently

$$
0=\frac{\mathrm{d}}{\mathrm{d} t} \dot{\mathbf{B}} \mathbf{B}^{\mathrm{T}}=\ddot{\mathbf{B}} \mathbf{B}^{\mathrm{T}}+\dot{\mathbf{B}} \dot{\mathbf{B}}^{\mathrm{T}}
$$

From Eq. (48) and (49), it is deduced

$$
\ddot{\mathbf{B}} \mathbf{B}^{\mathrm{T}}=-\dot{\mathbf{B}} \dot{\mathbf{B}}^{\mathrm{T}}=-\dot{\mathbf{B}} \mathbf{B}^{\mathrm{T}} \mathbf{B} \dot{\mathbf{B}}^{\mathrm{T}}=-\Omega^{2}(\tilde{\mathbf{G}}+\tilde{\mathbf{J}})(\tilde{\mathbf{G}}+\tilde{\mathbf{J}})^{\mathrm{T}} .
$$

Further analysis shows that $\tilde{\mathbf{G}}$ is antisymmetric, and Eq. (50) can be written as

$$
\ddot{\mathbf{B}} \mathbf{B}^{\mathrm{T}}=-\dot{\mathbf{B}} \dot{\mathbf{B}}^{\mathrm{T}}=-\dot{\mathbf{B}} \mathbf{B}^{\mathrm{T}} \mathbf{B} \dot{\mathbf{B}}^{\mathrm{T}}=\Omega^{2}(\tilde{\mathbf{G}}+\tilde{\mathbf{J}})(\tilde{\mathbf{G}}+\tilde{\mathbf{J}}) .
$$

The generalised force of the Eq. (35) is, from Eq. (32)

$$
\mathbf{Q}_{\mathrm{q}}(t)=\mathbf{B} \mathbf{Q}_{\mathrm{p}}(t)=\mathbf{B} \iiint_{\text {Volume }} \mathbf{\Phi}\left(\mathbf{A}(t)^{\mathrm{T}} \mathbf{v}\right)^{\mathrm{T}} \mathbf{A}(t)^{\mathrm{T}} \mathbf{g}^{v}(\mathbf{v}, t) \mathrm{d} v .
$$

By substituting Eq. (13) in Eq. (52), it is found

$$
\mathbf{Q}_{\mathrm{q}}(t)=\mathbf{B} \iiint_{\text {Volume }} \mathbf{B}^{\mathrm{T}} \boldsymbol{\Phi}(\mathbf{v})^{\mathrm{T}} \mathbf{g}^{v}(\mathbf{v}, t) \mathrm{d} v=\iiint_{\text {Volume }} \boldsymbol{\Phi}(\mathbf{v})^{\mathrm{T}} \mathbf{g}^{v}(\mathbf{v}, t) \mathrm{d} v .
$$

In standard cases, the vector associated with external volume forces can be written as

$$
\mathbf{g}^{v}(\mathbf{v}, t)=\mathbf{F}^{v}(\mathbf{v}) \gamma(t),
$$

and the generalised force expression is

$$
\mathbf{Q}_{\mathrm{q}}(t)=\iiint_{\text {Volume }} \boldsymbol{\Phi}(\mathbf{v})^{\mathrm{T}} \mathbf{F}^{v}(\mathbf{v}) \mathrm{d} v \gamma(t)=\tilde{\mathbf{F}} \gamma(t) .
$$

Now Eqs. (36)-(40), (48), (51) and (55) are substituted into Eq. (35), yielding

$$
\ddot{\mathbf{q}}+(\tilde{\mathbf{D}}-2 \Omega \tilde{\mathbf{G}}) \dot{\mathbf{q}}+\left(\tilde{\mathbf{K}}+\Omega^{2} \tilde{\mathbf{C}}-\Omega \tilde{\mathbf{D}}(\tilde{\mathbf{G}}+\tilde{\mathbf{J}})\right) \mathbf{q}=\tilde{\mathbf{F}} \gamma(t)+\Omega^{2} \tilde{\mathbf{L}},
$$

where

$$
\tilde{\mathbf{C}}=\tilde{\mathbf{G}} \tilde{\mathbf{G}}+\tilde{\mathbf{G}} \tilde{\mathbf{J}}-\tilde{\mathbf{J}} \tilde{\mathbf{G}}-\tilde{\mathbf{J}} \tilde{\mathbf{J}}-\tilde{\mathbf{E}}
$$

It must be drawn attention to the fact that the matrices $\widetilde{\mathbf{D}}, \widetilde{\mathbf{G}}, \widetilde{\mathbf{J}}, \widetilde{\mathbf{K}}, \widetilde{\mathbf{E}}, \widetilde{\mathbf{C}}, \widetilde{\mathbf{F}}$ and $\tilde{\mathbf{L}}$ are constant matrices and they are computed at the beginning of the simulation. An advantage of this Eulerian approach is that, if external forces do not rotate with the solid, the generalised force vector in Eulerian coordinates $\mathbf{Q}_{\mathrm{q}}(t)$ is calculated by integrating the volume of the solid independently of time. Therefore, it may be also 
calculated at the beginning of the simulation, unlike in the Lagrangian approach where the generalised force term $\mathbf{Q}_{\mathrm{p}}(t)$ must be evaluated at each time instant by using a costly integration over the volume. Moreover, the equation of motion (56) is linear and it can be adopted for obtaining the FRFs associated with forces or displacements that are applied or measured at fixed spatial points.

Finally, it is worth noting that Eq. (56) is expressed in a non-rotating reference frame whereas the Lagrangian methods such as Finite Element Method or Eq. (34) are based in a floating frame.

\section{Application of the method to a simply supported beam}

The parameters of the beam that are considered in the model are the radius $R$, length $L$, Young's modulus $E$, cross-sectional area $A$ and second moment of area $I$. In order to find a parallelism between the proposed method and beam theories, in the following the variables of the rotating Rayleigh beam formulation which are presented in the Appendix are adopted.

If $N$ bending modes of the Rayleigh beam are consider, the matrix of mode shapes $\boldsymbol{\Phi}$ becomes

$$
\boldsymbol{\Phi}=\left[\begin{array}{ccccc}
\phi_{1}(z) & 0 & \cdots & \phi_{N}(z) & 0 \\
0 & \phi_{1}(z) & \cdots & 0 & \phi_{N}(z) \\
-x \frac{\mathrm{d} \phi_{1}}{\mathrm{~d} z} & -y \frac{\mathrm{d} \phi_{1}}{\mathrm{~d} z} & \cdots & -x \frac{\mathrm{d} \phi_{N}}{\mathrm{~d} z} & -y \frac{\mathrm{d} \phi_{N}}{\mathrm{~d} z}
\end{array}\right]
$$

where $\phi_{j}$ is computed from Eq. (A.1). Taking into account the modal gyroscopic term $g_{j}$ defined in (A.8) and the modal functions detailed in Eq. (58), the matrices that define the equation of motion for rotating solids, Eq. (56), become

$$
\begin{gathered}
\tilde{\mathbf{G}}=\left[\begin{array}{ccccc}
0 & -g_{1} & \cdots & 0 & 0 \\
g_{1} & 0 & \cdots & 0 & 0 \\
\vdots & \vdots & \ddots & \vdots & \vdots \\
0 & 0 & \cdots & 0 & -g_{N} \\
0 & 0 & \cdots & g_{N} & 0
\end{array}\right], \\
\tilde{\mathbf{J}}=\left[\begin{array}{ccccc}
0 & -\left(1-g_{1}\right) & \cdots & 0 & 0 \\
\left(1-g_{1}\right) & 0 & \cdots & 0 & 0 \\
\vdots & \vdots & \ddots & \vdots & \vdots \\
0 & 0 & \cdots & 0 & -\left(1-g_{N}\right) \\
0 & 0 & \cdots & \left(1-g_{N}\right) & 0
\end{array}\right],
\end{gathered}
$$




$$
\begin{gathered}
\widetilde{\mathbf{E}}=\left[\begin{array}{ccccc}
\left(1-g_{1}\right) & 0 & \cdots & 0 & 0 \\
0 & \left(1-g_{1}\right) & \cdots & 0 & 0 \\
\vdots & \vdots & \ddots & \vdots & \vdots \\
0 & 0 & \cdots & \left(1-g_{N}\right) & 0 \\
0 & 0 & \cdots & 0 & \left(1-g_{N}\right)
\end{array}\right], \\
\tilde{\mathbf{L}}=\mathbf{0},
\end{gathered}
$$

and

$$
\tilde{\mathbf{C}}=\left[\begin{array}{ccccc}
-g_{1} & 0 & \cdots & 0 & 0 \\
0 & -g_{1} & \cdots & 0 & 0 \\
\vdots & \vdots & \ddots & \vdots & \vdots \\
0 & 0 & \cdots & -g_{N} & 0 \\
0 & 0 & \cdots & 0 & -g_{N}
\end{array}\right] .
$$

The matrices of Eqs. (59)-(63) uncouple the influence between the modes in Eq. (56). Therefore, Eq. (56) can be written for each pair of modal coordinates associated with the $j$-th mode $\mathbf{q}^{j}$, that is

$$
\ddot{\mathbf{q}}^{j}+2\left(\Omega g_{j} \mathbf{G}+c_{j} \mathbf{I}\right) \dot{\mathbf{q}}^{j}+\left(\left(\omega_{j}^{2}-\Omega^{2} g_{j}\right) \mathbf{I}+2 \Omega c_{j} \mathbf{G}\right) \mathbf{q}^{j}=\mathbf{Q}^{j},
$$

where all the terms are defined in the Appendix. Eq. (64) is identical to Eq. (A.6) with the exception of the term $-\Omega^{2} g_{j}$ that accounts for the effect of the centrifugal forces when the section is deformed. The one-dimensional Rayleigh beam model does not consider this effect and therefore, some differences are expected in the results. Further discussion will be provided in Section 5 .

The eigenvalues of the Eq. (64) are

$$
\begin{gathered}
\lambda_{j, 1}=-c_{j}+i g_{j} \Omega+\sqrt{c_{j}^{2}-\omega_{j}^{2}+g_{j} \Omega^{2}-g_{j}^{2} \Omega^{2}-2 i \Omega c_{j}\left(g_{j}-1\right)}, \\
\lambda_{j, 2}=-c_{j}+i g_{j} \Omega-\sqrt{c_{j}^{2}-\omega_{j}^{2}+g_{j} \Omega^{2}-g_{j}^{2} \Omega^{2}-2 i \Omega c_{j}\left(g_{j}-1\right)}, \\
\lambda_{j, 3}=\lambda_{j, 1}^{*}, \\
\lambda_{j, 4}=\lambda_{j, 2}^{*} .
\end{gathered}
$$

Instable behaviour happens when the first eigenvalue in Eq. (65) has positive real part. It is then concluded that the critical speed is

$$
\Omega_{c r}=\frac{\omega_{k}}{\sqrt{1-g_{k}}},
$$

such critical velocity being lower than that predicted by the one-dimensional beam model (A.11). 


\section{Computational method}

In general, an analytical expression of the mode shapes cannot be obtained and consequently, numerical methods like FE are used to compute the modal properties. The present section presents a method for obtaining the matrices of the equation of motion in Eq. (56) from the FE modal solution.

FE modal analysis provides the modal solution in the nodes of the solid mesh. The modal matrix $\boldsymbol{\Phi}_{F E}$ is obtained with the FE model, whose columns contain the values given by $\boldsymbol{\Phi}(\mathbf{u})$ on each node.

The modal function in the domain of an $e$-th element can be interpolated as

$$
\boldsymbol{\Phi}(\mathbf{u})=\mathbf{N}_{e}(\mathbf{u}) \boldsymbol{\Phi}_{F E}
$$

where the matrix $\mathbf{N}_{e}(\mathbf{u})$ contains the shape functions (or basis) of the $e$-th element.

Bearing in mind the nodal interpolation defined in (67), the matrices of the equation of motion in Eulerian coordinates, Eq. (56), are shown below

$$
\begin{gathered}
\tilde{\mathbf{J}}=\iiint_{\text {Volume }} \rho \boldsymbol{\Phi}^{\mathrm{T}} \mathbf{J} \boldsymbol{\Phi} \mathrm{d} v=\sum_{e=1}^{n_{e}} \iiint_{V_{e}} \rho \boldsymbol{\Phi}^{\mathrm{T}} \mathbf{J} \boldsymbol{\Phi} \mathrm{d} v=\boldsymbol{\Phi}_{F E}^{\mathrm{T}}\left(\sum_{e=1}^{n_{e}} \iiint_{V_{e}} \rho \mathbf{N}_{e}{ }^{\mathrm{T}} \mathbf{J} \mathbf{N}_{e} \mathrm{~d} v\right) \boldsymbol{\Phi}_{F E}, \\
\widetilde{\mathbf{E}}=\boldsymbol{\Phi}_{F E}^{\mathrm{T}}\left(\sum_{e=1}^{n_{e}} \iiint_{V_{e}} \rho \mathbf{N}_{e}^{\mathrm{T}} \mathbf{E} \mathbf{N}_{e} \mathrm{~d} v\right) \boldsymbol{\Phi}_{F E}, \\
\tilde{\mathbf{L}}=\boldsymbol{\Phi}_{F E}^{\mathrm{T}}\left(\sum_{e=1}^{n_{e}} \iiint_{V_{e}} \rho \mathbf{N}_{e}{ }^{\mathrm{T}} \mathbf{E} \mathbf{u} \mathrm{d} v\right), \\
\tilde{\mathbf{G}}=\boldsymbol{\Phi}_{F E}^{\mathrm{T}}\left(\sum_{e=1}^{n_{e}} \iiint_{V_{e}} \rho\left(\sum_{i=1}^{3} \frac{\partial \mathbf{N}_{e}(\mathbf{u})^{\mathrm{T}}}{\partial u_{i}}(\mathbf{J} \mathbf{u})_{i}\right) \mathbf{N}_{e} \mathrm{~d} v\right) \boldsymbol{\Phi}_{F E},
\end{gathered}
$$

where $n_{e}$ is the number of elements in the FE mesh, and $V_{e}$ is the volume of the $e$-th element.

\section{Results}

This section analyses the stability, modal properties and FRF of the simply suported rotating flexible cylinder with internal damping. The aim of the present section is to compare the results from three different approaches: first, the Rayleigh rotating beam model (in the following, the beam model) whose formulas can be found in the Appendix of the present paper, the proposed method in which the modes are computed analytically (analytical solid model), and the proposed method where the computations are performed numerically (numerical solid model). 
The present study considers the first bending mode, being the forward mode the unstable mode (further details can be found in [3] and will be also shown later). The properties of the simply supported cylinder are defined in Table 1 . The first three natural frequencies associated with bending modes are given in Table 2. The cylinder mesh considered in the numerical solid model is depicted in Fig. 2. The FE model implements tridimensional lineal elements.

Table 1. Cylinder properties.

\begin{tabular}{ll}
\hline Length & $L=1.5 \mathrm{~m}$ \\
Radius & $R=0.05 \mathrm{~m}$ \\
Density & $\rho=7800 \mathrm{~kg} / \mathrm{m}^{3}$ \\
Young's modulus & $E=2.1 \times 10^{11} \mathrm{~N} / \mathrm{m}^{2}$ \\
Internal damping ratio & $\xi=0.03$ \\
Poisson's ratio & $v=0.3$ \\
\hline
\end{tabular}

Table 2. Natural frequencies of the cylinder [Hz].

\begin{tabular}{llll}
\hline Mode number & Mode type & FE & Rayleigh beam model \\
\hline 1 & 1st bending mode & 90.14 & 90.44 \\
2 & 2nd bending mode & 355.21 & 360.27 \\
3 & 3rd bending mode & 780.51 & 805.17 \\
\hline
\end{tabular}

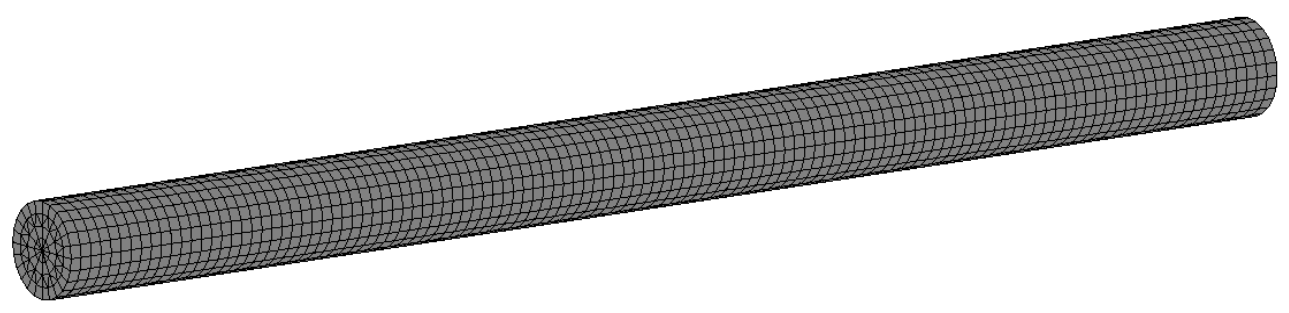

Fig. 2. FE mesh of the cylinder.

Fig. 3 plots the eigenvalues in the Argand diagram for the three formulations previously indicated. These have been calculated with a spin speed sweep, reaching almost twice the critical speed. The differences between the models are analysed in the following figures. Fig. 4a shows the real part of the eigenvalues. All the models describe virtually the same evolution of the real part of the eigenvalues. In addition, it is clearly shown that the real part of the backward eigenvalue is always negative and therefore stable, while the real part of the eigenvalue associated with the forward mode is positive beyond critical speed and hence becomes unstable. For the forward mode, 
Fig. 4b presents the real part of the associated eigenvalue against the ratio angular velocity/forward natural frequency. The instability of the forward mode takes place if the angular velocity is higher than the forward mode frequency, in the absence of external damping as in the case under analysis.

The critical speed is now shown in Fig. 5 for several internal damping ratios $\xi$. The critical velocity does not depend on the internal damping rate in the absence of external damping. These results are in accordance with the conclusions of rotating shaft literature [3].

The discrepancies between the models are associated with the imaginary part of the eigenvalues as can be seen in Fig. 6. The evolution of the natural frequency with the angular velocity (Campbell diagram) for the three formulations is shown. The analytical and numerical solid models predict virtually the same evolution, but differ in comparison with the beam model. As can be seen, this discrepancy increases with spin speed. Although the difference between models is less than $0.5 \%$, the proposed solid model describes a very different evolution and trend compared with the beam model. 


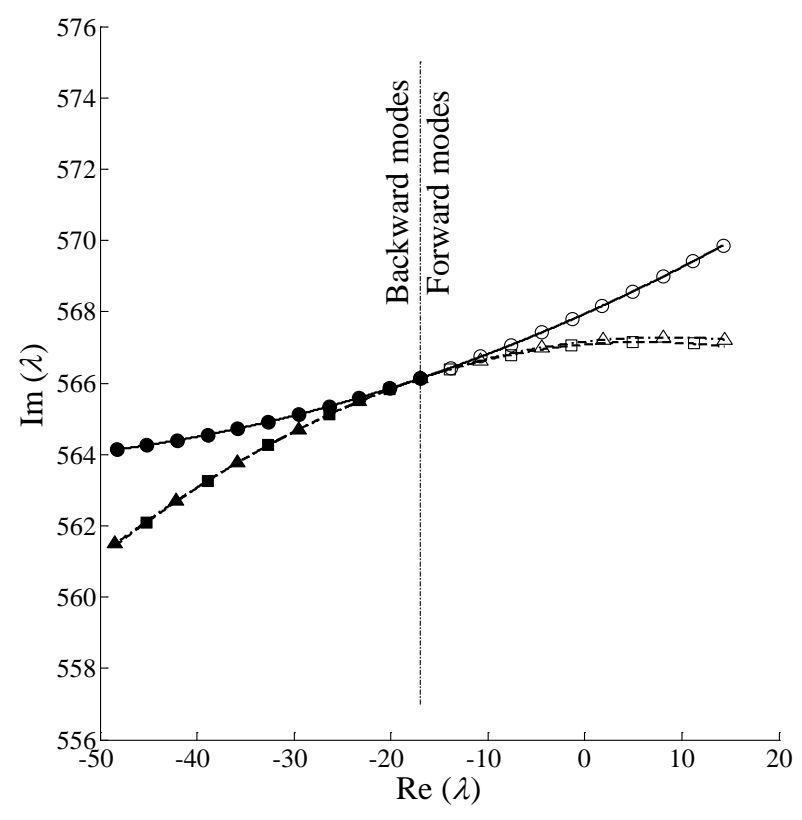

Fig. 3. Real and imaginary part of the eigenvalues: $\bullet,-\bullet$, forward and backward modes,

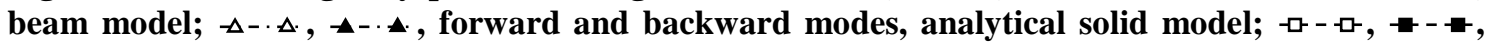
forward and backward modes, numerical solid model.

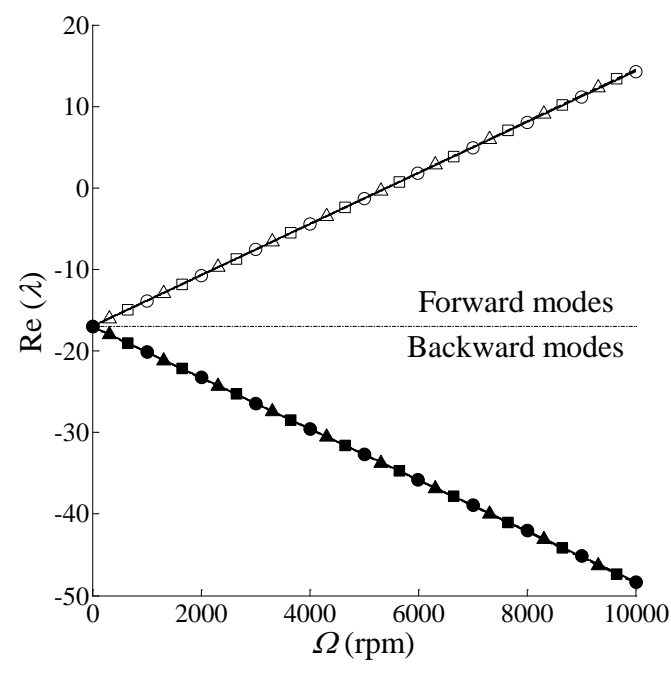

a)

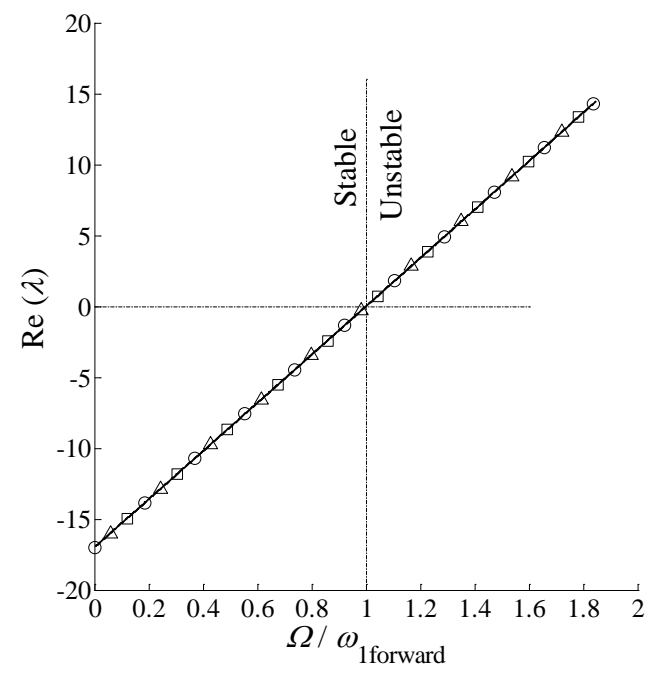

b)

Fig. 4. Real part of the eigenvalues: $\bullet-\bullet-\bullet$, forward and backward modes, beam model;

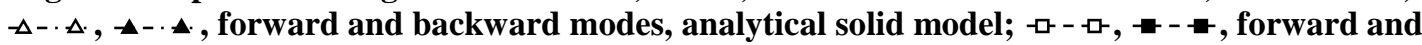
backward modes, numerical solid model. 


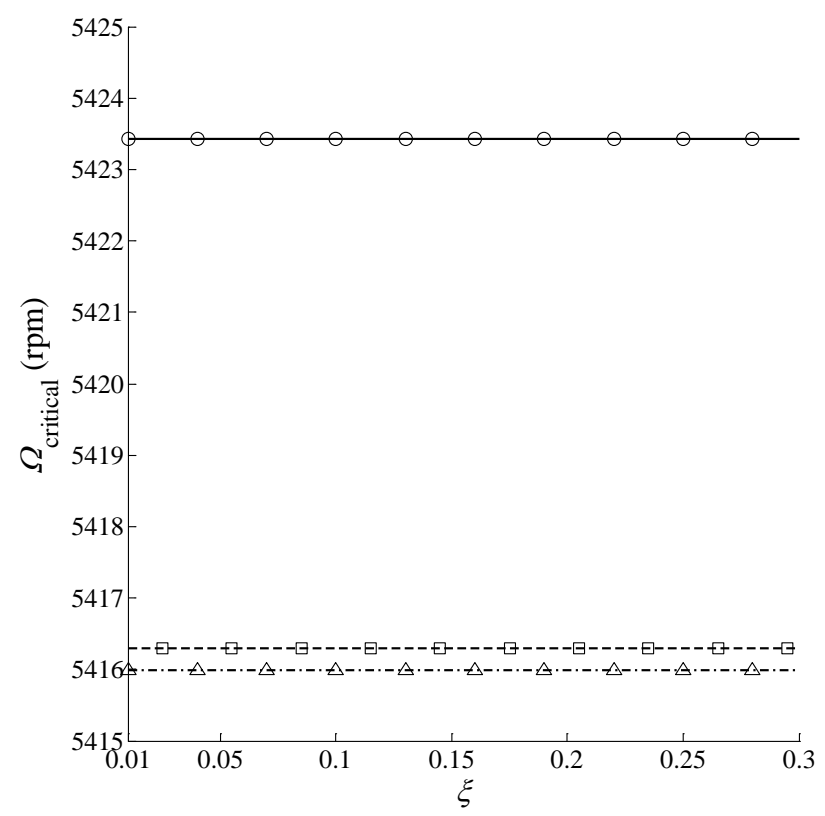

Fig. 5. Critical speed for different internal damping ratios: $\bullet-\odot$, beam model; $-\Delta_{-} \cdot \Delta^{-}$, analytical solid model; $-\square--\square$, numerical solid model.

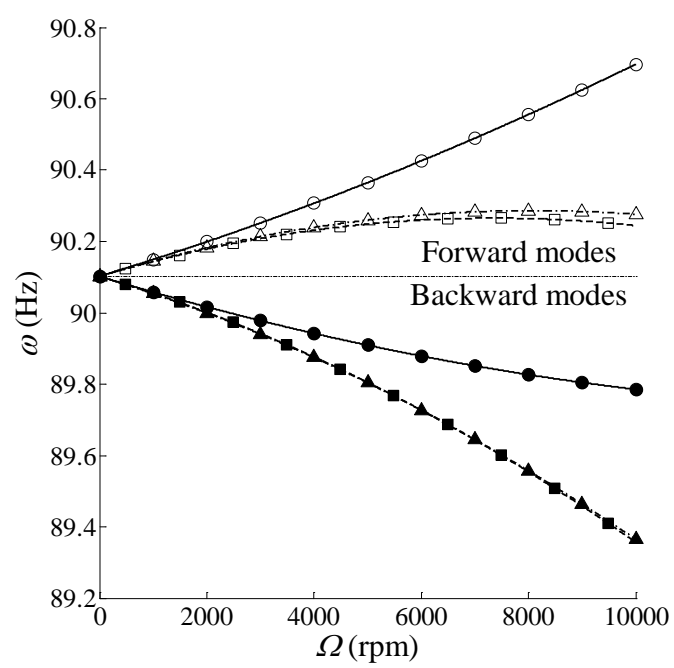

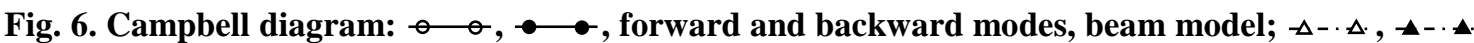
, forward and backward modes, analytical solid model; $-\square_{--},--_{---}$, forward and backward modes, the numerical solid model.

In the introduction section of this article, it was stated that one advantage of the proposed solid model is to allow the calculation of FRF associated with forces or displacements that are applied or measured at fixed spatial points. The FRF for the simply supported cylinder is shown in Fig. 11 for different internal damping ratios; the force is applied transversely in the central node of the cylinder, and the displacement is measured at the same point and same direction. It can be seen that the backward 
bending mode is highly damped and disappears for $\xi=0.01$. In this way, the steady response is only influenced by the forward bending mode. The same case is calculated through the three formulations for $\xi=0.03$ and the associated FRFs are shown in Fig. 8. As observed previously, the analytical and numerical solid models predict the same behaviour with undistinguishable curves, but they differ from the beam model. It is worth noting that the backward mode has disappeared, remaining only the forward bending mode.

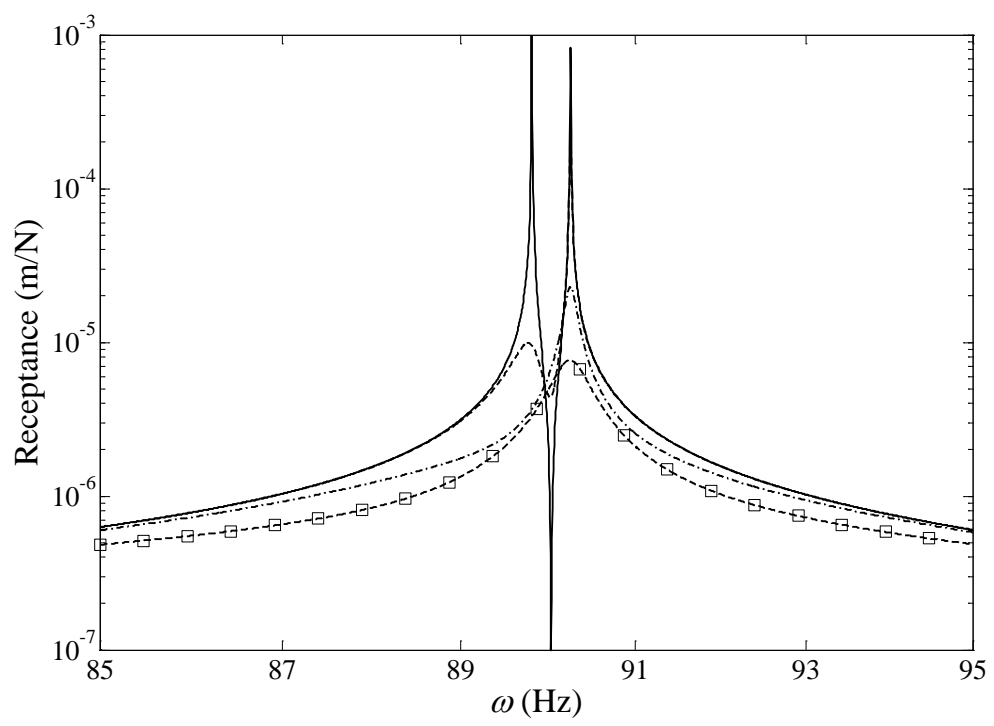

Fig. 7. Direct FRF (receptance) calculated, numerical solid model at $\Omega=5000 \mathrm{rpm}$ for different internal damping ratios: $\longrightarrow, \xi=0 ; \ldots,-., \xi=0.001 ; \ldots \ldots, \xi=0.01 ;-\square-\square, \xi=0.03$.

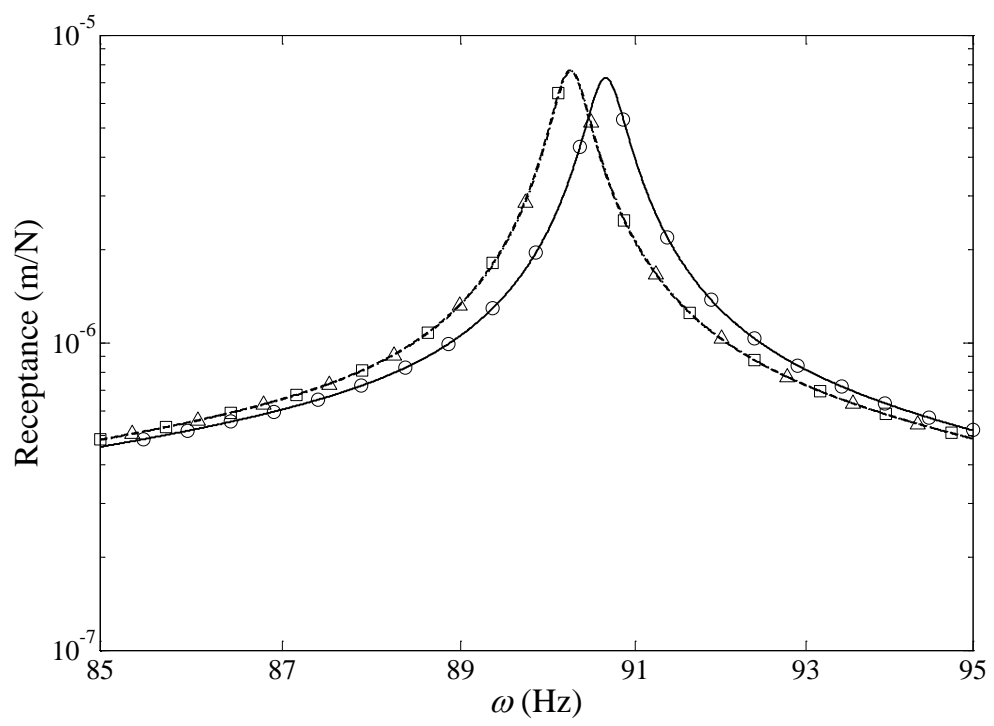

Fig. 8. Direct FRF (receptance) for $\Omega=5000 \mathrm{rpm}$ and $\xi=0.03:-\mathrm{C}^{-}$, beam model; $-\Delta-\Delta$, analytical solid model; - - - - - , numerical solid model. 
It is important to emphasise the effect done by the centrifugal forces associated with the deformation, being the fundamental difference between the proposed solid model and the one-dimensional beam model. This effect is represented by the term $\Omega^{2} g_{j}$ for the analytical solid model in Eq. (64) or its equivalent $\Omega^{2} \widetilde{\mathbf{C}}$ in the solid model from Eq. (56), and it does not appear in the beam model. The proposed model by means of analytical data produces the same equation of motion in Eq. (64) than the rotating beam model in Eq. (A.6) if the term $\Omega^{2} g_{j}$ is neglected in the former model. Therefore, the following calculations show the results from the rotating Rayleigh beam model and the proposed numerical solid model where the term $\Omega^{2} \widetilde{\mathbf{C}}$ is omitted.

In the previous section, the solid model showed not only the discrepancies in the imaginary part but also a quite different tendency in comparison with the beam model. Once neglected the effect done by the centrifugal forces associated with the solid deformation, both models describe a very similar evolution of the natural frequency, as shown in Fig. 9.

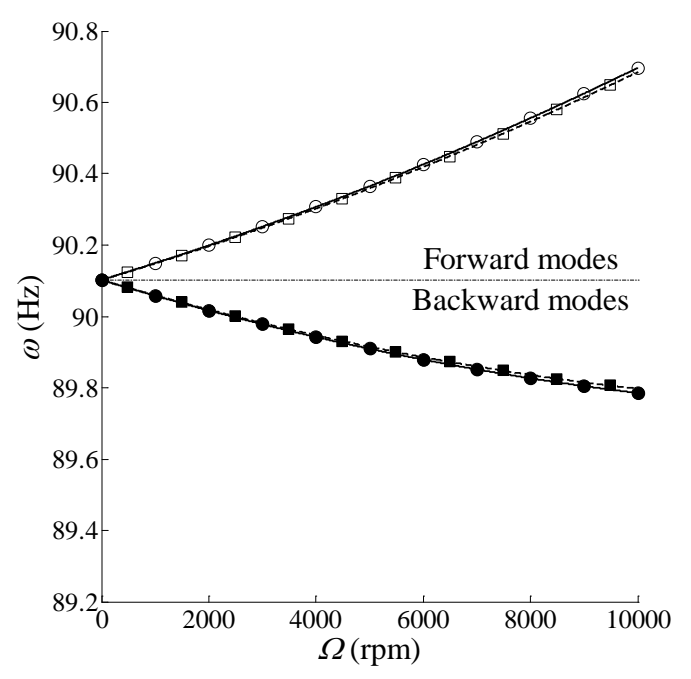

Fig. 9. Campbell diagram: $\bullet \bullet, \bullet \bullet-$, forward and backward modes, beam model; $-\square--\square,-\rightarrow$ , forward and backward modes, numerical solid model.

Finally, Fig. 10 shows the FRF when the influence of the centrifugal forces is neglected. As can be seen the predictions associated with both models are almost undistinguishable, with overlapped FRF curves in the frequency range considered. 


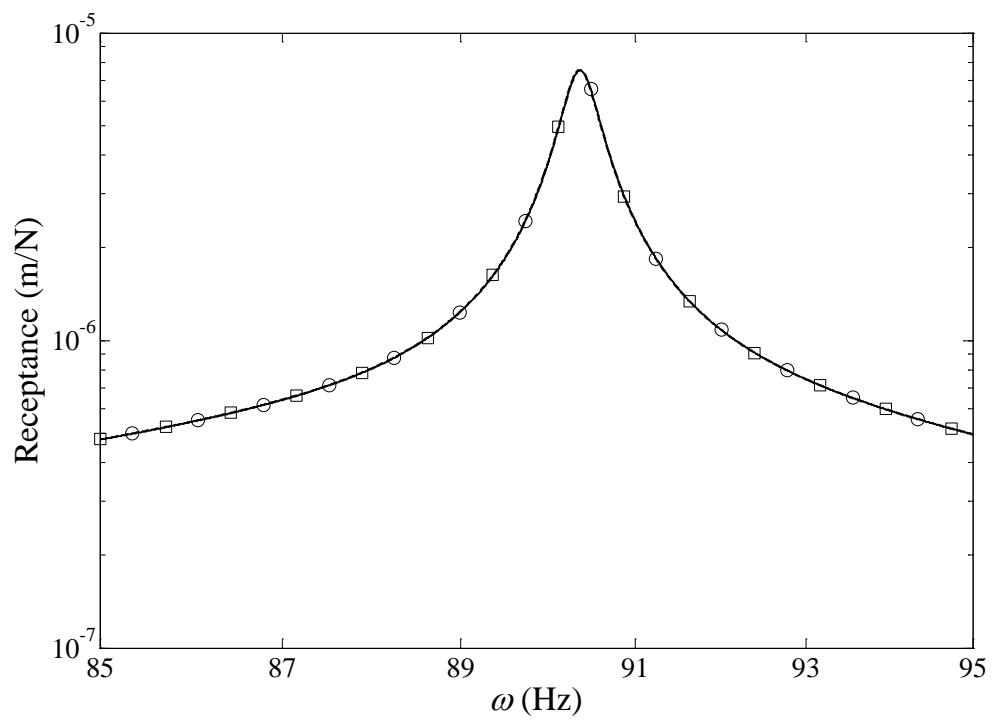

Fig. 10. Direct FRF (receptance) for $\Omega=5000 \mathrm{rpm}$ and $\xi=0.03$. ๑ $\bullet$, beam model; $-\square-\square$, numerical solid model.

\section{Conclusions}

A complete methodology to model the dynamics of damped elastic solids of revolution rotating about their axis of revolution has been developed in this article. This model is designed for those cases in which the interest lies in spatial points of the solid rather than material points. Therefore, it is especially suitable for complex systems consisting of rotating and non-rotating solids that are in mutual interaction.

The method is based on a modal approach where the modal properties of the non-rotating solid form the modal basis of the system. The final formulation consists of a set of linear ordinary differential equations where the coefficients are time independent. Therefore the matrices are calculated only once at the beginning of the simulation, leading to a considerable reduction of the computational cost.

The proposed method can be applied to the study of the stability, to obtain the equivalent modal properties of the rotating solid and also to compute the FRFs of the solid. Two versions of the method have been presented: analytical consideration of the modal properties of the solid (as a Rayleigh beam), and numerical implementation from FE data. In addition, these two approaches are compared with a one-dimensional Rayleigh beam model. It has been shown that the analytical and numerical solid models predict virtually the same dynamic behaviour of the case under study. However, the proposed solid model shows discrepancies with the Rayleigh beam model. These discrepancies are due to the effect of the centrifugal forces associated with the deformed shape of the solid, which is only considered in the proposed solid model, represented by the terms $-\Omega^{2} g_{j}$ and $\Omega^{2} \tilde{\mathbf{C}}$ in Eqs. (64) and (56) respectively.

Finally, in order to describe the dynamic behaviour of a beam by means of the proposed solid model, the terms $-\Omega^{2} g_{j}$ or $\Omega^{2} \widetilde{\mathbf{C}}$ have been eliminated. In this case, 
the calculations show that the proposed solid model behaves like the Rayleigh beam model (see Figs. 13-19).

\section{Acknowledgement}

The authors gratefully acknowledge the support for this work provided by the Project TRA2010-15669 (Ministerio de Ciencia e Innovación).

\section{Appendix}

In this appendix, the formulation associated with the one-dimensional simply supported Rayleigh beam model, including rotation and internal modal viscous damping is summarised. Further details can be found in Ref. [3].

In accordance with the frame of reference in Fig. 1, the $j$-th mass normalised bending mode calculated in the neutral axis of Rayleigh beam is

$$
\phi_{j}(z)=\frac{1}{\sqrt{m_{j}}} \sin \left(\frac{j \pi z}{L}\right)
$$

and the $j$-th natural frequency is

$$
\omega_{j}=\sqrt{\frac{k_{j}}{m_{j}}},
$$

where the constants $k_{j}$ and $m_{j}$ have the following expressions

$$
\begin{gathered}
k_{j}=\frac{E I(j \pi)^{4}}{2 L^{3}}, \\
m_{j}=\frac{\rho A L}{2}+\frac{\rho I(j \pi)^{2}}{2 L} .
\end{gathered}
$$

The transverse displacements are computed from the fixed frame through the following modal approach

$$
\left\{\begin{array}{l}
x(z, t) \\
y(z, t)
\end{array}\right\}=\sum_{j=1}^{\infty} \phi_{j}(z)\left\{\begin{array}{c}
q_{x}^{j}(t) \\
q_{y}^{j}(t)
\end{array}\right\}=\sum_{j=1}^{\infty} \phi_{j} \mathbf{q}^{j},
$$


where $\mathbf{q}^{j}$ is the two-dimensional vector with modal coordinates associated with the $j$-th orthogonal bending mode shape. The equation of motion for the damped simply supported rotating Rayleigh beam in modal coordinates is

$$
\ddot{\mathbf{q}}^{j}+2\left(\Omega g_{j} \mathbf{G}+c_{j} \mathbf{I}\right) \dot{\mathbf{q}}^{j}+\left(\omega_{j}^{2} \mathbf{I}+2 \Omega c_{j} \mathbf{G}\right) \mathbf{q}^{j}=\mathbf{Q}^{j},
$$

$\mathbf{Q}^{j}$ being the generalised external force, $\mathbf{I}$ is the identity matrix $2 \times 2, \Omega$ is the beam spin speed and $\omega_{j}$ is the natural frequency of the $j$-th bending mode of the simply supported non-rotating Rayleigh beam [17-19]. The term $c_{j}$ is computed from the internal modal damping ratio of the $j$-th bending mode $\xi_{j}$ as

$$
c_{j}=\omega_{j} \xi_{j}
$$

The modal gyroscopic term $g_{j}$ is obtained from the following expression

$$
g_{j}=\frac{(j \pi)^{2}}{\zeta^{2}+(j \pi)^{2}},
$$

where $g_{j}$ is, by definition, bounded between 0 and 1 , and $\zeta$ is the slenderness of the beam. The matrix $\mathbf{G}$ is anti-symmetric and couples both orthogonal bending modes. It can be expressed as

$$
\mathbf{G}=\left(\begin{array}{cc}
0 & 1 \\
-1 & 0
\end{array}\right)
$$

The eigenvalues of the Eq. (A.6) are

$$
\begin{gathered}
\lambda_{j, 1}=-c_{j}+i g_{j} \Omega+\sqrt{c_{j}^{2}-\omega_{j}^{2}-g_{j}^{2} \Omega^{2}-2 i \Omega c_{j}\left(g_{j}-1\right)} \\
\lambda_{j, 2}=-c_{j}+i g_{j} \Omega-\sqrt{c_{j}^{2}-\omega_{j}^{2}-g_{j}^{2} \Omega^{2}-2 i \Omega c_{j}\left(g_{j}-1\right)} \\
\lambda_{k, 3}=\lambda_{k, 1}^{*} \\
\lambda_{k, 4}=\lambda_{k, 2}^{*}
\end{gathered}
$$

where the notation $z^{*}$ represents the complex conjugate of $z, \lambda_{k, 1}$ and $\lambda_{k, 3}$ are the eigenvalues for the forward mode, and $\lambda_{k, 2}$ and $\lambda_{k, 4}$ are associated with the backward mode. The forward modes reveal a critical speed beyond which the real part of their eigenvalues is positive. The modes become then unstable (a conclusion also made in $[20,21])$ beyond the critical angular velocity given by

$$
\Omega_{c r}=\frac{\omega_{1}}{\sqrt{1-2 g_{1}}}
$$




\section{References}

[1] B.L. Newkirk, Shaft whipping, General Electric Review 27 (1924) 169-178.

[2] A.L. Kimball, Internal friction theory of shaft whirling. General Electric Review 27 (1924) 224-251.

[3] G. Genta, Dynamics of Rotating Systems, Springer, New York, 2005.

[4] C.W. Lee, Vibration Analysis of Rotors, Kluwer, Dordrecht, 1993.

[5] H.D. Nelson, J.M. McVaugh, The dynamics of rotor-bearing systems using finite element. Journal of Engineering for Industry 98 (1976) 593-600.

[6] E.S. Zorzi, H.D. Nelson, Finite element simulation of rotor-bearing systems with internal damping. Journal of Engineering for Power 99 (1977) 71-76.

[7] L.W. Chen, D.M. Ku, Analysis of whirl speeds of rotor-bearing systems with internal damping by $\mathrm{C}^{\circ}$ finite elements. Finite Elements in Analysis and Design 9 (1991) 169-176.

[8] D. Ku, Finite element analysis of whirl speeds for rotor-bearing systems with internal damping. Mechanical Systems and Signal Processing 12 (1998) 599-610.

[9] R. Sino, E. Chatelet, O. Montagnier, G. Jacquet-Richardet, Dynamic instability analysis of internally damped rotors, Proceedings of ASME Turbo Expo 2007 Power for Land, Sea and Air, Vol. 5, Montréal, May 2007, pp. 727-736.

[10] M.A. Hili, T. Fakhfakh, M. Haddar, Vibration analysis of a rotating flexible shaftdisk system. Journal of Engineering Mathematics 57 (2007) 351-363.

[11] G. Jacquet-Richardet, G. Ferraris, P. Rieutord, Frequencies and modes of rotating flexible bladed disc-shaft assemblies: a global cyclic symmetry approach. Journal of Sound and Vibration 191 (1996) 901-915.

[12] J. Yu, A. Craggs, 3-D solid finite element modeling of rotating shafts, Proceedings of the 15th International Modal Analysis Conference - Imac, Vols I and II, Vol. 3089, Orlando, 1997, pp. 1488-1494.

[13] E. Chatelet, D. Lornage, G. Jacquet-Richardet, A three dimensional modeling of the dynamic behavior of composite rotors. International Journal of Rotating Machinery 8 (2002) 185-192.

[14] J. Fayos, L. Baeza, F.D. Denia, J.E. Tarancón, An Eulerian coordinate-based method for analysing the structural vibrations of a solid of revolution rotating about its main axis. Journal of Sound and Vibration 306 (2007) 618-635.

[15] M.A. Brown, A.A. Shabana, Application of multibody methodology to rotating shaft problems. Journal of Sound and Vibration 204 (1997) 439-457.

[16] A.A. Shabana, Dynamics of Multibody Systems (Third Edition), Cambridge University Press, 2005.

[17] A.A. Shabana, Theory of Vibration, Vol. II, Discrete and Continuous Systems, Springer-Verlag, New York, Inc., 1991.

[18] W. Weaver Jr., S.P. Timoshenko, D.H. Young, Vibration Problems in Engineering (Fifth Edition), Wiley, New York, 1990.

[19] G. Genta, Vibration of Structures and Machines, Practical Aspects (Third Edition), Springer, New York, 1999. 
[20] J. Melanson, J.W. Zu, Free vibration and stability analysis of internally damped rotating shafts with general boundary conditions. Journal of Vibration and Acoustic 120 (1998) 776-783.

[21] G.J. Sheu, S.M. Yang, Dynamic analysis of a spinning Rayleigh beam. International Journal of Mechanical Sciences 47 (2005) 157-169. 